\title{
Training Load and Carbohydrate Periodization Practices of Elite Male Australian Football Players: Evidence of Fueling for the Work Required
}

\author{
Harry E. Routledge \\ Liverpool John Moores University and Port Adelaide Football Club \\ Rocco Di Michele \\ University of Bologna
}

\author{
Stuart Graham \\ Port Adelaide Football Club \\ Darren Burgess \\ Port Adelaide Football Club
}

\author{
Robert M. Erskine, Graeme L. Close, and James P. Morton \\ Liverpool John Moores University
}

\begin{abstract}
The authors aimed to quantify (a) the periodization of physical loading and daily carbohydrate (CHO) intake across an in-season weekly microcycle of Australian Football and (b) the quantity and source of CHO consumed during game play and training. Physical loading (via global positioning system technology) and daily CHO intake (via a combination of 24-hr recall, food diaries, and remote food photographic method) were assessed in 42 professional male players during two weekly microcycles comprising a home and away fixture. The players also reported the source and quantity of $\mathrm{CHO}$ consumed during all games ( $n=22$ games) and on the training session completed 4 days before each game ( $n=22$ sessions). The total distance was greater $(p<.05)$ on game day $(\mathrm{GD} ; 13 \mathrm{~km})$ versus all training days. The total distance differed between training days, where GD-2 $(8 \mathrm{~km})$ was higher than GD-1, GD-3, and GD-4 (3.5, 0, and $7 \mathrm{~km}$, respectively). The daily CHO intake was also different between training days, with reported intakes of 1.8, 1.4, 2.5, and $4.5 \mathrm{~g} / \mathrm{kg}$ body mass on GD-4, GD-3, GD-2, and GD-1, respectively. The $\mathrm{CHO}$ intake was greater $(p<.05)$ during games $(59 \pm 19 \mathrm{~g})$ compared with training $(1 \pm 1 \mathrm{~g})$, where in the former, $75 \%$ of the $\mathrm{CHO}$ consumed was from fluids as opposed to gels. Although the data suggest that Australian Football players practice elements of $\mathrm{CHO}$ periodization, the low absolute $\mathrm{CHO}$ intakes likely represent considerable underreporting in this population. Even when accounting for potential underreporting, the data also suggest Australian Football players underconsume CHO in relation to the physical demands of training and competition.
\end{abstract}

Keywords: GPS, sports drinks, sports gels

The match activity profile data of Australian Football (AF) are well documented (Coutts et al., 2010; Dawson et al., 2004; Wiseby et al., 2010) and are greater than those observed in other invasive field team sports, such as soccer and rugby league (Varley et al., 2014). Given the high-intensity intermittent nature of AF match play and training, it follows that carbohydrate $(\mathrm{CHO})$ and muscle glycogen are likely the predominant substrate for energy production (Routledge et al., 2019). Accordingly, the current CHO recommendations for invasive team sports advise high $\mathrm{CHO}$ availability $(>6 \mathrm{~g} / \mathrm{kg})$ in the day prior to match play (Burke et al., 2011) and an intake of 30-60 g/hr during match play to maximize physical, technical, and cognitive performance (Burke et al., 2011).

In contrast to ensuring high $\mathrm{CHO}$ availability before and during competition, accumulating data over the last decade suggest that deliberately completing carefully selected training sessions

Routledge, Erskine, Close, and Morton are with the Research Institute for Sport and Exercise Sciences, Liverpool John Moores University, Liverpool, United Kingdom. Routledge, Graham, and Burgess are with Port Adelaide Football Club, Allan Scott Headquarters, Alberton, South Australia, Australia. Di Michele is with the Department of Biomedical and Neuromotor Sciences, University of Bologna, Bologna, Italy. Morton (J.P.Morton@ @ ljmu.ac.uk) is corresponding author. with reduced $\mathrm{CHO}$ availability may lead to greater adaptive responses of skeletal muscle (Bartlett et al., 2015; Hawley \& Morton, 2014; Impey et al., 2018). Indeed, the concept of "fueling for the work required" has recently been suggested as a practical framework for which to apply nutritional periodization strategies to both endurance (Impey et al., 2016, 2018) and team sport athletes (Anderson et al., 2017). Such strategies are intended to promote components of training adaptation (e.g., activation of regulatory cell-signalling pathways), but yet, also ensure adequate $\mathrm{CHO}$ (and energy) availability to promote competitive performance, reduce injury risk, and aid recovery (Burke et al., 2011).

We have recently reported that professional rugby league (Bradley et al., 2016), rugby union (Bradley et al., 2015), and soccer players (Anderson et al., 2017) habitually adjust daily CHO intake in accordance with the upcoming workload. To the authors' knowledge, however, the habitual $\mathrm{CHO}$ feeding strategies of AF players across weekly microcycles comprising both game play and training sessions have not yet been reported. With this in mind, the aims of the present study were to quantify (a) the periodization of physical loading and daily $\mathrm{CHO}$ intake across an in-season weekly microcycle of $\mathrm{AF}$ and (b) the quantity and source of $\mathrm{CHO}$ consumed during game play versus training. 


\section{Methods}

\section{Subjects}

Forty-two professional AF players from an AF list (mean $\pm S D$ : age $24 \pm 3$ years, height: $188 \pm 7.8 \mathrm{~cm}$, and body mass: $85 \pm 8.1 \mathrm{~kg}$ ) volunteered to take part in the study. The study was conducted according to the Declaration of Helsinki and was approved by the Ethics Committee of Liverpool John Moores University.

\section{Study Design}

The data collection was conducted across the entire 2017 Australian Football League Premiership season. In a repeated-measures design, the players reported their habitual daily $\mathrm{CHO}$ intake during two weekly microcycles comprising a back-to-back home and away fixture during the midseason period of June 2017. For each fixture, the players reported their daily $\mathrm{CHO}$ intake for the 4 days prior to each game. In addition, the players also reported CHO consumed during each game of the season ( $n=22$ games), as well as the highest loading training session of each training week across the season ( $n=22$ sessions), the latter occurring 2 days before each game. For game day (GD), the 22 selected players were studied, whereas during training sessions, the full 42-man squad was studied. The players studied had not been exposed to any formal educational training by the club's staff on CHO periodization strategies or messaging (e.g., fueling for the work required), though players had previously received training on the importance of "CHO loading" for game play. In addition, the types of foods and fluids available to the players while present under the club's care (e.g., training ground) did not change from day to day during the study period.

\section{Reported Daily Energy and Macronutrient Intake}

Daily energy intake was assessed from a 4-day food diary (using household measures to estimate portion sizes). Food diaries also contained educational pictures of portion sizes to assist players with self-reporting daily energy intakes. On the day prior to data collection, food diaries were explained to the players by the lead researcher, and an initial dietary habits questionnaire (24-hr food recall) was also completed. These questionnaires were used to establish habitual eating patterns and, subsequently, allowed for a follow-up analysis of food diaries, as they helped to retrieve any potential information that players may have missed in their food diary input. In addition to food diaries, each player also completed the remote food photographic method, in which they sent time-stamped photographs each day (via Whatsapp messaging service) to the lead researcher (Martin et al., 2009). The food diaries were cross-checked using a 24-hr recall with the lead researcher after 1 day of entries. In this way, the lead researcher used the information obtained from the three approaches to dietary assessment (i.e., food diaries, 24-hr recall, and the remote food photographic method) to collectively estimate self-reported daily energy intake, an approach used previously with team sport athletes (Anderson et al., 2016). Energy intake and macronutrient composition were assessed from dietary analysis software (Nutritics Ltd, Dublin, Ireland). To assist with entering portion sizes into the dietary analysis software, the players' photographs of single source foods (e.g., rice, bread, fruit, chicken, etc.) and composite meals (e.g., spaghetti bolognese, lasagne, etc.) were cross-referenced against visual databases (www.carbsandcals.com, London, UK).

\section{Reported Carbohydrate Intake During Training and Games}

The $\mathrm{CHO}$ consumed during training and games was also reported by the players within $30 \mathrm{~min}$ after the physical weekly training session ( $n=22$ sessions) on GD minus 2 (GD-2) and within 30 min after each game ( $n=22$ games). The $\mathrm{CHO}$ intake during training and games was quantified according to dietary recall, after prior education on reporting quantities and source. The players had unrestricted access to water, sports drinks, and $\mathrm{CHO}$ gels during both training and match play, where each player was initially provided with their own individually labeled sports drink and water bottles (both $500 \mathrm{ml}$ ). If the players ran out of either bottle, another was then prepared by the lead researcher. Prior to the data collection period (preseason), the players were instructed on the correct method of recalling $\mathrm{CHO}$ intake during training and games. During six preseason training sessions with the lead researcher present, the players had unrestricted access to water, sports drinks (36 g CHO, 33 g sucrose, and 3 g glucose; Gatorade, Chatswood, NSW, Australia), and energy gels (29.8 g CHO and 2.9 g sugars; Shotz, Sydney, NSW, Australia). Following each session, the lead researcher would demonstrate the correct method to report $\mathrm{CHO}$ intake with the players present. This was repeated six times across the preseason prior to the start of data collection (Round 1 of the 2017 Australian Football League season season). For the data collection period following each game and training session, the players would use a record sheet that was observed by the lead researcher. Furthermore, during matches, the lead researcher was present on the team bench and had visible access to crossreference player recall data. The lead researcher was also present for all training sessions and had full access to the players to visibly crossreference player recall.

\section{Quantification of Training and Game Load}

In-season external training load and match activity profiles were quantified using a portable global positioning system microtechnology device (Optimeye S5; Catapult Innovations, Melbourne, VIC, Australia). At the conclusion of each training session and competitive match, data were downloaded and analyzed using the manufacturer specific software (Catapult Openfield software, version 11.1.2; Catapult Innovations). The data were filtered using the manufacturer's "Intelligent Motion Filter" to remove erroneous global positioning system information. Satellite data sampled at $10 \mathrm{~Hz}$ provided measures of total distance covered and distance covered relative to specific velocity bands corresponding to (Zone 1, 0-1.5 m/s; Zone 2, 1.5-3 m/s; Zone 3, 3-4.16 m/s; Zone 4, 4.16-5.5 m/s; Zone 5, 5.5-7 m/s; and Zone $6,>7 \mathrm{~m} / \mathrm{s}$ ). For the purposes of this study, the relative velocity bands 4-6 were assigned a qualitative descriptor, Zone 4, "Running"; Zone 5, "High-Speed Running"; and Zone 6, "Sprinting."

\section{Statistical Analysis}

Statistical analysis was conducted using the Statistical Package for Social Sciences software program (SPSS, version 17; IBM, Armonk, NY). External load and macronutrient content were analyzed using a two-way repeated-measure General Linear Model, where the within-subject factors were day (GD-4, GD-3, GD-2, and GD-1) and fixture week (Home and Away). All data in the text, figures, and tables are presented as mean $\pm S D$, with $p$ values $<.05$ indicating statistical significance. 


\section{Results}

\section{Assessment of External Training Load}

The external load metrics for the home and away microcycle are presented in Table 1. The external load was higher (all $p \mathrm{~s}<.01$ ) on GD compared with GD-4, GD-3, GD-2, and GD-1 for total distance, running, high-speed running, and sprinting. Specifically, GD-2 displayed higher loading $(p<.01)$ than GD-4, GD-3, and GD-1 for total distance and running on GD-3 and GD-1, although there was no difference between GD-4 $(p=.06)$. High-speed running and sprinting were also greater on GD-2 $(p<.01)$ than GD-4, GD-3, and GD-1. There were no differences in the external loads between the home and away games.

\section{Self-Reported Daily Energy and Macronutrient Intakes}

The self-reported daily energy and macronutrient intake are presented in Table 2. Daily energy and absolute and relative CHO intake were not significantly different between the home and away fixture schedules ( $p=.19, p=.48$, and $p=.23$, respectively). Daily absolute and relative $\mathrm{CHO}$ intake were significantly different between days (all $p \mathrm{~s}<.001$ ), such that significant differences were evident between all pairwise comparisons (all $p s<.001$ ). The players reported the lowest daily energy and $\mathrm{CHO}$ intake on GD-3 (i.e., the day when no training was performed), whereas the highest intakes were reported on GD-1 (i.e., the day before the game). Although the reported absolute and relative protein (both $p \mathrm{~s}<.001$ ) and fat intakes (both $p \mathrm{~s}<.001$ ) were also significantly different between days, they did not follow the same pairwise comparisons as the reported energy and CHO intakes (see Table 2 for location of specific differences). The players also reported consuming more protein and less fat during home fixture schedules compared with away fixture schedules $(p=.002$ and $p=.005$, respectively).

\section{Reported Pregame CHO Intake}

Reported absolute $(p=.8)$ and relative CHO intake $(p=.7)$ consumed in the prematch meal was not significantly different between the home and away game (see Figure 1a and 1b).

\section{Reported CHO Intake During Training and Games}

Carbohydrates consumed during game play $(59 \pm 19 \mathrm{~g})$ was higher $(p<.05)$ than that consumed during training sessions $(1 \pm 1 \mathrm{~g}$; Figure 1c). Accordingly, $\mathrm{CHO}$ intake during exercise when expressed as gram per hour $(34 \pm 11 \mathrm{~g} / \mathrm{hr}$ vs. $1 \pm 1 \mathrm{~g} / \mathrm{hr}$; Figure $1 \mathrm{~d})$ and gram per minute $(0.6 \pm 0.2 \mathrm{~g} / \mathrm{min}$ vs. $0.01 \pm 0.1 \mathrm{~g} / \mathrm{min}$; Figure 1e) was also higher $(p<.05)$ during game play compared with training. In the context of game play, $53 \%$ of the athletes achieved CHO intakes in the recommended range of 30-60 g/hr, with $30 \%$ consuming $<30 \mathrm{~g} / \mathrm{hr}$ and $17 \%$ consuming $>60 \mathrm{~g} / \mathrm{hr}$. During game play, a greater amount of $\mathrm{CHO}$ was consumed from fluids versus gels $(p<.05)$ when expressed as total CHO $(38 \pm 18 \mathrm{~g}$ vs. $22 \pm 20 \mathrm{~g})$ in gram per hour $(21 \pm 24 \mathrm{~g} / \mathrm{hr}$ vs. $13 \pm 14 \mathrm{~g} / \mathrm{hr})$ and gram per minute $(0.4 \pm 0.2 \mathrm{~g} / \mathrm{min}$ vs. $0.2 \pm 0.2 \mathrm{~g} / \mathrm{min})$.

\section{Discussion}

Using a seasonal long analysis, we report for the first time that (a) "in-season" AF training sessions exhibit marked reductions in physical load compared with game play, (b) players' reported daily $\mathrm{CHO}$ intake varies during the weekly microcycle, and (c) players consume greater amounts of $\mathrm{CHO}$ (predominantly from fluid sources) during game play versus training. Altogether, these data suggest that players adjust their daily $\mathrm{CHO}$ intake in accordance with periodization of the weekly loading patterns. However, an evaluation of reported daily $\mathrm{CHO}$ intake suggests that players are likely underconsuming $\mathrm{CHO}$, especially when considered in the context of $\mathrm{CHO}$ loading for match play.

Table 1 Total Distance, Distance Covered While Running (4.16-5.5 m/s), High-Speed Running (5.5-7 m/s), Sprinting (>7 m/s), and Session Duration During a Weekly Microcycle Comprising a Home and Away AF Fixture

\begin{tabular}{|c|c|c|c|c|c|}
\hline Fixture & GD-4 & GD-3 & GD-2 & GD-1 & GD \\
\hline \multicolumn{6}{|c|}{ Total distance $(\mathrm{m})$} \\
\hline Home & $7,644 \pm 578 * * *(59 \%)$ & $0 \pm 0 * * *(0 \%)$ & $7,568 \pm 437 *(59 \%)$ & $3,588 \pm 278 * * *(28 \%)$ & $12,900 \pm 1,152(100 \%)$ \\
\hline Away & $6,667 \pm 511 * * *(50 \%)$ & $0 \pm 0 * * *(0 \%)$ & $8,084 \pm 497 *(60 \%)$ & $3,603 \pm 260 * * *(27 \%)$ & $13,372 \pm 1,378(100 \%)$ \\
\hline \multicolumn{6}{|c|}{ Running (m) } \\
\hline Home & $1,389 \pm 348 *(52 \%)$ & $0 \pm 0 * * *(0 \%)$ & $1,050 \pm 195^{*}(40 \%)$ & $540 \pm 136 * * *(20 \%)$ & $2,650 \pm 592(100 \%)$ \\
\hline Away & $862 \pm 182 *(33 \%)$ & $0 \pm 0 * * *(0 \%)$ & $1,436 \pm 282 *(55 \%)$ & $534 \pm 132 * * *(21 \%)$ & $2,626 \pm 567(100 \%)$ \\
\hline \multicolumn{6}{|c|}{ High-speed running (m) } \\
\hline Home & $624 \pm 190 * * *(64 \%)$ & $0 \pm 0 * * *(0 \%)$ & $907 \pm 216 *(93 \%)$ & $228 \pm 82 * * *(23 \%)$ & $977 \pm 341(100 \%)$ \\
\hline Away & $427 \pm 106^{* * * *}(44 \%)$ & $0 \pm 0 * * *(0 \%)$ & $714 \pm 161 *(74 \%)$ & $251 \pm 155^{*} * *(26 \%)$ & $962 \pm 333(100 \%)$ \\
\hline \multicolumn{6}{|c|}{ Sprinting (m) } \\
\hline Home & $70 \pm 49 * * *(48 \%)$ & $0 \pm 0 * * *(0 \%)$ & $123 \pm 54 *(84 \%)$ & $25 \pm 33 * * *(17 \%)$ & $146 \pm 121(100 \%)$ \\
\hline Away & $103 \pm 44 * * *(69 \%)$ & $0 \pm 0 * * *(0 \%)$ & $97 \pm 60 *(65 \%)$ & $24 \pm 30 * * *(16 \%)$ & $149 \pm 116(100 \%)$ \\
\hline \multicolumn{6}{|c|}{ Session duration (min) } \\
\hline Home & $78 \pm 3(75 \%)$ & $0 \pm 0(0 \%)$ & $83 \pm 0(79 \%)$ & $40 \pm 2(38 \%)$ & $104 \pm 9(100 \%)$ \\
\hline Away & $78 \pm 5(71 \%)$ & $0 \pm 0(0 \%)$ & $81 \pm 0(74 \%)$ & $41 \pm 3(37 \%)$ & $110 \pm 14(100 \%)$ \\
\hline
\end{tabular}

Note. Data in parentheses represent data expressed as a percentage of the game play load. AF = Australian Football; GD = game day.

$*$ Significance from GD, $p<.05$. **Significance from GD-2, $p<.05$. 
Table 2 Total Energy, Absolute and Relative CHO, Protein, and Fat Intakes During a Weekly Microcycle Comprising a Home and Away AF Fixture

\begin{tabular}{|c|c|c|c|c|}
\hline Fixture & GD-4 & GD-3 & GD-2 & GD-1 \\
\hline \multicolumn{5}{|c|}{ Energy (kcal) } \\
\hline Home & $2,606 \pm 80$ & $2,344 \pm 104 * * * *$ & $2,981 \pm 471 * * *$ & $3,559 \pm 584 * * *, * * *$ \\
\hline Away & $2,586 \pm 105$ & $2,347 \pm 99 * * * * *$ & $3,119 \pm 443 * * *$ & $3,597 \pm 573 * * * * * * *$ \\
\hline \multicolumn{5}{|l|}{$\mathrm{CHO}(\mathrm{g})$} \\
\hline Home & $190 \pm 7$ & $135 \pm 21 * * * * *$ & $221 \pm 10^{* * * *}$ & $389 \pm 54 *, * *, * * *$ \\
\hline Away & $164 \pm 30$ & $126 \pm 16^{* * * * *}$ & $224 \pm 11 * * *$ & $428 \pm 47 *, * *, * * *$ \\
\hline \multicolumn{5}{|c|}{$\mathrm{CHO}(\mathrm{g} / \mathrm{kg})$} \\
\hline Home & $2.1 \pm 0.2$ & $1.5 \pm 0.2 * * * *$ & $2.5 \pm 0.3 * * *$ & $4.3 \pm 0.8 * * * * * * *$ \\
\hline Away & $1.8 \pm 0.3$ & $1.4 \pm 0.2 * * * *$ & $2.5 \pm 0.2 * * *$ & $4.8 \pm 0.8 * * * * * * *$ \\
\hline \multicolumn{5}{|c|}{ Protein (g) } \\
\hline Home & $232 \pm 29$ & $190 \pm 47 *$ & $252 \pm 26^{* * * *}$ & $188 \pm 43 * * * * *$ \\
\hline Away & $226 \pm 35$ & $175 \pm 42 *$ & $234 \pm 24 * * *$ & $190 \pm 45 * * * *$ \\
\hline \multicolumn{5}{|c|}{ Protein $(\mathrm{g} / \mathrm{kg})$} \\
\hline Home & $2.6 \pm 0.3$ & $2.1 \pm 0.6^{*}$ & $2.8 \pm 0.4 * * *$ & $2.1 \pm 0.5 * * * *$ \\
\hline Away & $2.5 \pm 0.5$ & $1.9 \pm 0.5^{*}$ & $2.6 \pm 0.3 * * *$ & $2.1 \pm 0.5^{* * * * *}$ \\
\hline \multicolumn{5}{|l|}{ Fat $(g)$} \\
\hline Home & $102 \pm 13$ & $116 \pm 12 *$ & $121 \pm 14^{* * * *}$ & $139 \pm 32 * * *$ \\
\hline Away & $114 \pm 19$ & $127 \pm 11 *$ & $143 \pm 17 * * *$ & $125 \pm 24 * * *$ \\
\hline \multicolumn{5}{|l|}{ Fat $(\mathrm{g} / \mathrm{kg})$} \\
\hline Home & $1.1 \pm 0.2$ & $1.3 \pm 0.2 *$ & $1.3 \pm 0.2 * * *$ & $1.5 \pm 0.4 * * *$ \\
\hline Away & $1.3 \pm 0.2$ & $1.4 \pm 0.2 *$ & $1.6 \pm 0.3^{* * * *}$ & $1.4 \pm 0.3^{* * * *}$ \\
\hline
\end{tabular}

Note. $\mathrm{GD}=$ game day; $\mathrm{AF}=$ Australian Football; $\mathrm{CHO}=$ carbohydrate.

*Significant difference from GD-4. **Significant difference from GD-3. ***Significant difference from GD-2.

To the authors' knowledge, we are the first group to quantify physical loading of multiple training sessions as opposed to weekly accumulative loads (Ritchie et al., 2016). We report that physical loads of training sessions were significantly less than game play, as quantified according to the total distance in both the home and away fixture weeks. The training session data are in conjunction with previous authors (Bartlett et al., 2017) who highlighted training session distance to be $6,389 \pm 3,315 \mathrm{~m}$. Total running, high-speed running, and sprinting metrics were all lower during training compared with games. Nonetheless, the distances covered during running $(1,400 \mathrm{~m})$ and high-speed running $(550 \mathrm{~m})$ in $\mathrm{AF}$ training are greater than the absolute distances observed in soccer (Anderson et al., 2016), where values of 500 and $100 \mathrm{~m}$ were reported, respectively. Such differences between codes are perhaps a reflection of the greater duration of training sessions in $\mathrm{AF}$ versus the training sessions observed by Anderson et al. (2016) (i.e., approximately 20 min longer), although we acknowledge this hypothesis is limited to the data provided for only two professional clubs. However, the present data clearly illustrate the periodization of training load across a weekly training microcycle.

In accordance with the evidence of training load periodization, our data also highlight practices of nutritional periodization, whereby a greater amount of $\mathrm{CHO}(5 \mathrm{~g} / \mathrm{kg})$ was consumed on the day prior to game play compared with lower relative $\mathrm{CHO}$ intakes (i.e., $<3 \mathrm{~g} / \mathrm{kg}$ ) on training days. The $\mathrm{CHO}$ intake reported here on GD-1 agree with previous data, where AF players reported consuming an average of $4.1 \mathrm{~g} \pm 1.6 \mathrm{~g} / \mathrm{kg} \mathrm{CHO}$ per day, as estimated from a 24-hr dietary recall (Bilsborough et al., 2016). However, the low $\mathrm{CHO}$ intakes reported on the remainder of the training days do not seem conducive to supporting the daily energetic requirement of male athletes (ranging from 80 to $90 \mathrm{~kg}$ ), even when considering rest days. As such, we acknowledge the potential of underreporting as a limitation of the present study. Support for the hypothesis of specifically underreporting $\mathrm{CHO}$ is provided by the observation that similar periodization patterns of daily energy and $\mathrm{CHO}$ intake were observed (see Table 1), whereas daily fat and protein intake did not follow similar patterns. When considering that daily protein and fat intakes were also within the recommended ranges for elite athletes, our data therefore suggest that players may be specifically underreporting $\mathrm{CHO}$ intakes.

In an attempt to account for the magnitude of potential underreporting (e.g., 10-45\%) previously observed in athletic populations (Magkos \& Yannakoulia, 2003), the range of players' self-reported CHO intakes on GD-1 can be recalculated to $5.3-6.9 \mathrm{~g} / \mathrm{kg}$, a range that is somewhat closer to the $\mathrm{CHO}$ loading guidelines (i.e., $7-12 \mathrm{~g} / \mathrm{kg}$ ) advised for the $24 \mathrm{hr}$ prior to competition. Nonetheless, even when accounting for such magnitudes of underreporting of daily $\mathrm{CHO}$ intake on the remaining training days (i.e., GD-4, GD-3, and GD-2), recalculation of players' $\mathrm{CHO}$ intake is still $<3.6 \mathrm{~g} / \mathrm{kg}$. Such intakes may be considered suboptimal when compared with the $\mathrm{CHO}$ guidelines (i.e., 5-7 $\mathrm{g} / \mathrm{kg}$ ) for athletes performing moderate-intensity training for 1-2 hr per day (Thomas et al., 2016). When considered this way, our data therefore suggest that the current cohort of $\mathrm{AF}$ players is likely underconsuming $\mathrm{CHO}$ in relation to contemporary guidelines, even when accounting for underreporting.

In relation to the quantity of $\mathrm{CHO}$ consumed during exercise, we observed marked differences between training $(1 \pm 1 \mathrm{~g} / \mathrm{hr})$ and match play $(34 \pm 11 \mathrm{~g} / \mathrm{hr})$. This apparent difference between $\mathrm{CHO}$ intake during each activity is similar to that reported by professional soccer players of the English Premier League, where values 

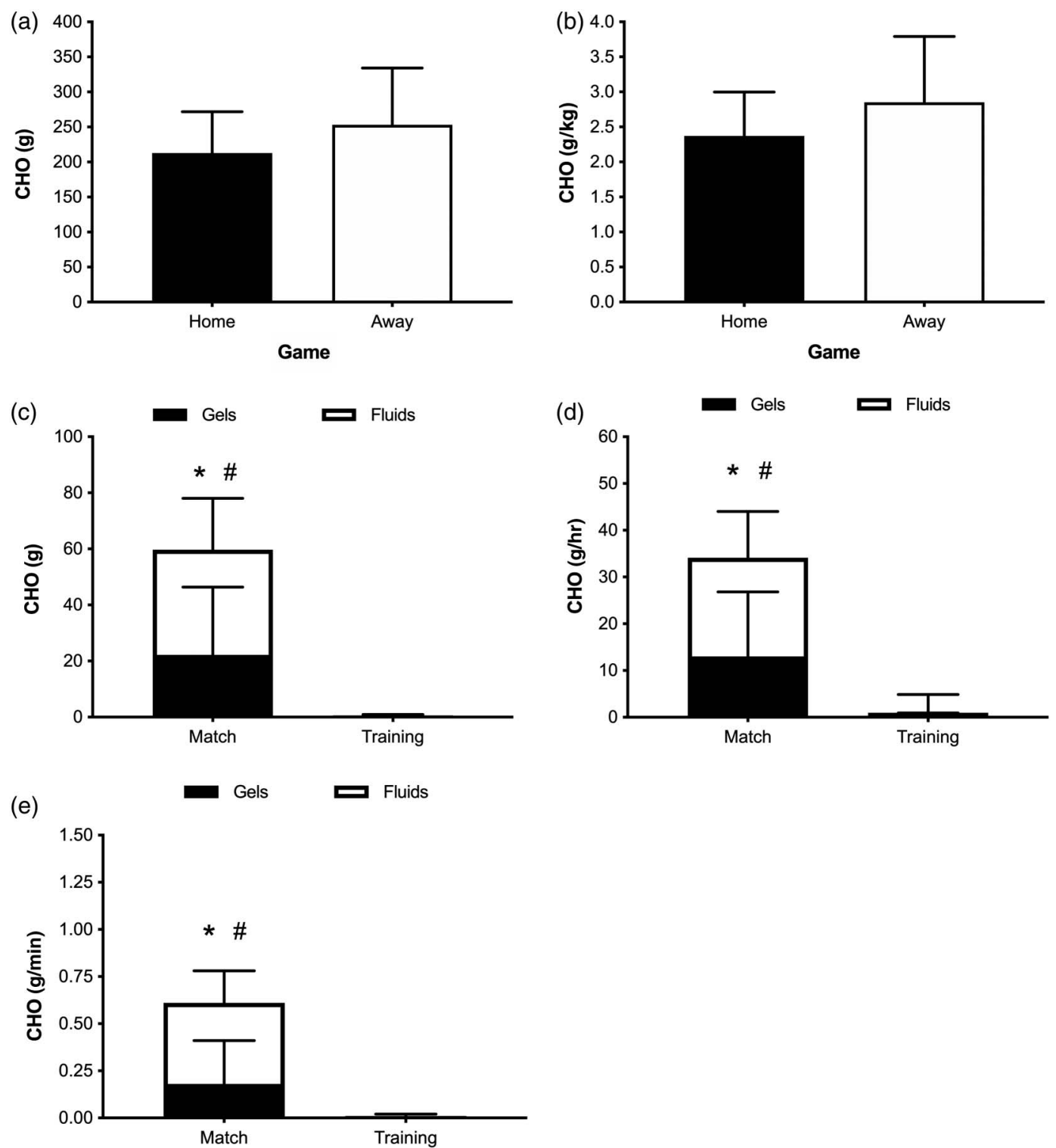

Figure 1 - Reported CHO intake in the prematch meal expressed as (a) absolute and (b) relative intake during a weekly microcycle comprising a home and away AF fixture. CHO consumed from fluids and gels during training and game play when expressed as (c) total CHO, (d) g/hr, and (e) g/min. *Significant difference between games and training, $p<.05$. "Significant differences between gels and fluids, $p<.05$. CHO = carbohydrate; $\mathrm{AF}=$ Australian Football.

of $3 \pm 4$ and $32 \pm 22 \mathrm{~g} / \mathrm{hr}$ were reported in training and match play, respectively (Anderson et al., 2017). In a case study account, we reported that consumption of $54 \mathrm{~g} / \mathrm{hr}$ of $\mathrm{CHO}$ during AF match play reduced muscle glycogen utilization compared with consumption of $0 \mathrm{~g} / \mathrm{hr}$ (Routledge et al., 2019). Of the athletes studied here, 53\% achieved CHO intakes in the recommended range of 30-60 g/hr. This finding contrasts with soccer players, where only $33 \%$ of the players consumed $>30 \mathrm{~g} / \mathrm{hr}$ (Anderson et al., 2017). Furthermore, although we reported that $63 \%$ of the $\mathrm{CHO}$ consumed by soccer players was from gel sources (Anderson et al., 2017), the athletes studied here consumed $75 \%$ of the $\mathrm{CHO}$ from fluid sources and only $25 \%$ from gel sources. Such differences between studies may be related to regular access to fluids and $\mathrm{CHO}$ gels during rotations in AF match play, as well as higher ambient temperatures observed during AF game play (as opposed to that typically observed during the annual English Premier League), potentially suggesting that players consciously practice a CHO feeding strategy that simultaneously intends to promote hydration. In contrast, the choice of gels may offer a superior strategy to English Premier League soccer players, as opposed to fluids per se (usually 6-8\% CHO solutions), owing to the flexibility for achieving $\mathrm{CHO}$ targets regardless of individual differences in body mass, hydration requirements, and differences in interchange number inherent to $\mathrm{AF}$.

It is difficult to ascertain if the low absolute $\mathrm{CHO}$ intake reported here and the apparent $\mathrm{CHO}$ periodization practices were a deliberate choice of the player or alternatively, an unconscious decision. All players had access to the same array of CHO foods and fluids during games and training, and they were not following any coach-led nutritional program, with the exception of receiving prior dietary education on increasing $\mathrm{CHO}$ portion sizes on the day before a game. Nonetheless, the $\mathrm{CHO}$ periodization practices reported here appear in accordance with the principle of "fuel 
for the work required" that suggests that athletes should adjust their daily CHO intake in accordance with the workload (Impey et al., 2018). When considered with our previous observations of professional rugby league (Morehen et al., 2016), rugby union (Bradley et al., 2015), and soccer players (Anderson et al., 2017), the present data contribute to the emerging evidence that team sport athletes habitually adjust both the total daily $\mathrm{CHO}$ intake and $\mathrm{CHO}$ intake during exercise in accordance with the upcoming workload. Nonetheless, even when accounting for potential underreporting of $\mathrm{CHO}$, the low $\mathrm{CHO}$ intakes reported here (considered indicative of underfueling) suggest that the current cohort of AF players would benefit from specific education on the $\mathrm{CHO}$ requirements of training and GDs. This is especially relevant when considering the training loads completed on GD-4 and GD-2, where total distances $>7 \mathrm{~km}$ were completed.

In summary, we simultaneously quantified for the first time the daily physical loading and habitual daily $\mathrm{CHO}$ intakes during two weekly in-season microcycles of elite-level AF players. When such data are considered in conjunction with the seasonal quantification of $\mathrm{CHO}$ intake during training and matches, our data demonstrate that AF players appear to practice elements of $\mathrm{CHO}$ periodization. Furthermore, although we acknowledge that the low absolute $\mathrm{CHO}$ intakes likely represent considerable underreporting in this population, the data also suggest that the current cohort of AF players underconsume $\mathrm{CHO}$ in relation to the physical demands of training and competition.

\section{References}

Anderson, L., Orme, P., Di Michelle, R., Close, G.L., Morgans, R., Drust, B., \& Morton, J.P. (2016). Quantification of training load during one-, two- and three game week schedules in professional soccer players from the English Premier League: Implication for carbohydrate periodisation. Journal of Sports Science, 34(13), 1250-1259. doi:10.1080/02640414.2015.1106574

Anderson, L., Orme, P., Naughton, R.J., Close, G.L., Milsom, J., Rydings, D., ... Morton, J.P. (2017). Energy intake and expenditure of professional soccer players of the English Premier League: Evidence of a carbohydrate periodisation. International Journal of Sport Nutrition and Exercise Metabolism, 27(3), 228-238.

Bartlett, J.D., Hawley, J.A., \& Morton, J.P. (2015). Carbohydrate availability and exercise training adaptation: Too much of a good thing? European Journal of Sports Science, 15(1), 3-12. doi:10.1080/ 17461391.2014.920926

Bartlett, J.D., O’Connor, F., Pitchford, N., Torres-Ronda, L., \& Robertson, S.J. (2017). Relationships between internal and external training load in team sports athletes: Evidence for an individualised approach. International Journal of Sports Physiology and Performance, 12(2), 230-234.

Bilsborough, J.C., Greenway, K., Livingston, S., Cordy, J., \& Coutts, A.J. (2016). Changes in anthropometry, upper-body strength and nutrient intake of professional Australian Rules Football players during a season. International Journal of Sports Physiology and Performance, 11(3), 290-300. PubMed ID: 26217046 doi:10.1123/ijspp.20140447

Bradley, W.J., Cavanagh, B., Douglas, W., Donovan, T.F., Twist, C., Morton, J.P., \& Close, G.L. (2015). Energy intake and expenditure assessed "in-season" in elite European rugby union squad. European Journal of Sport Science, 15(6), 469-479. PubMed ID: 26055695 doi:10.1080/17461391.2015.1042528
Bradley, W.J., Morehen, J.C., Haigh, J., Clarke, J., Donovan, T.F., Twist, C., ... Close, G.L. (2016). Muscle glycogen utilisation during Rugby match play: Effects of pre-game carbohydrate. Journal of Science and Medicine in Sport, 19(12), 1033-1038. PubMed ID: 27134132 doi:10.1016/j.jsams.2016.03.008

Burke, L.M., Hawley, J.A., Wong, S.H., \& Jeukendrup, A.E. (2011). Carbohydrates for training and competition. Journal of Sports Science, 29(1), 17-27. doi:10.1080/02640414.2011.585473

Coutts, A.J., Quinn, J., Hocking, J., Castagna, C., \& Rampinini, E. (2010). Match running performance in elite Australian Rules Football. Journal of Science and Medicine in Sport, 13(5), 543-548. PubMed ID: 19853508 doi:10.1016/j.jsams.2009. 09.004

Dawson, B., Hopkinson, R., Appleby, B., Stewart, G., \& Roberts, C. (2004). Player movement patterns and game activities in the Australian Football League. Journal of Science and Medicine in Sport, 7(3), 278-291. PubMed ID: 15518293 doi:10.1016/S1440-2440(04) 80023-9

Hawley, J.A., \& Morton, J.P. (2014). Ramping up the signal: Promoting endurance training adaptation in skeletal muscle by nutritional manipulation. Clinical and Experimental Pharmacology and Physiology, 41(8), 608-613. PubMed ID: 25142094 doi:10.1111/14401681.12246

Impey, S.G., Hammond, K.M., Shepherd, S.O., Sharples, A.P., Stewart, C., Limb, M., ... Morton, J.P. (2016). Fuel for the work required: A practical approach to amalgamating train-low paradigms for endurance athletes. Physiological Reports, 4(10), e12803.

Impey, S.G., Hearris, M.A., Hammond, K.M., Bartlett, J.D., Louis, J., Close, G.L., \& Morton, J.P. (2018). Fuel for the work required: A theoretical framework for carbohydrate periodization and the glycogen threshold hypothesis. Sports Medicine, 48(5), 1031-1048. PubMed ID: 29453741 doi:10.1007/s40279-0180867-7

Martin, C.K., Han, H., Coulson, S.M., Allen, H.R., Champagne, C.M., \& Anton, S.D. (2009). A novel method to remotely measure food intake of free-living individuals in real time: The remote food photography method. British Journal of Nutrition, 101(3), 446-456. PubMed ID: 18616837 doi:10.1017/S0007114508027438

Magkos, F., \& Yannakoulia, M. (2003). Methodology of dietary assessment in athletes: Concepts and pitfalls. Current Opinion in Clinical Nutrition and Metabolic Care, 6(5), 539-549. PubMed ID: 12913671 doi:10.1097/00075197-200309000-00007

Morehen, J.C., Bradley, W.J., Clarke, J., Twist, C., Hambly, C., Speakman, J.R., ... Close, G.L. (2016). The assessment of total energy expenditure during a 14-day in-season period of professional Rugby league players using the doubly labelled water method. International Journal of Sport Nutrition and Exercise Metabolism, 26(5), 464-472. PubMed ID: 27096279 doi:10. 1123/ijsnem.2015-0335

Ritchie, D., Hopkins, W.G., Buchheit, M., Cordy, J., \& Bartlett, J.D. (2016). Quantification of training and competition load across a season in an elite Australian football club. International Journal of Sports Physiology and Performance, 11(4), 474-479. PubMed ID: 26355304 doi:10.1123/ijspp.2015-0294

Routledge, H.E., Leckey, J.J., Lee, M.J., Garnham, A., Graham, S., Burgess, D., ... Morton, J.P. (2019). Muscle glycogen utilisation during an Australian Rules Football Game. International Journal of Sports Physiology and Performance,14, 122-124.

Thomas, D.T., Erdman, K.A., \& Burke, L.M. (2016). Position of the academy of the nutrition and dietetics, dietitians of Canada, and the American College of Sports Medicine: Nutrition and Athletic 
Performance. Journal of the Academy of Nutrition and Dietetics, 116(3), 501-528. PubMed ID: 26920240 doi:10.1016/j.jand.2015. 12.006

Varley, M.C., Gabbett, T., \& Aughey, R.J. (2014). Activity profiles of professional soccer, rugby league and Australian football match play. Journal of Sports Science, 32(20), 1858-1866. doi:10.1080/ 02640414.2013.823227

Wiseby, B., Montgomery, P.G., Pyne, D.B., \& Rattray, B. (2010). Quantifying movement demands of AFL football using GPS tracking. Journal of Science and Medicine in Sport, 13(5), 531-536. 